\title{
PEERS-Förderpreise in 5 Kategorien
}

Zum 8. Mal sind junge Wissenschaftler und Zahnärzte an Kliniken und in niedergelassenen Praxen aufgerufen, sich mit ihren Arbeiten zu aktuellen Themen der Implantologie um einen der PEERS-Förderpreise $\mathrm{zu}$ bewerben. In 5 verschiedenen Kategorien werden die mit 3000 bis $6000 €$ dotierten Preise für Arbeiten mit klinischer Relevanz vergeben: Fallpräsentation (Poster), wissenschaftliches Poster und wissenschaftliche Publikation. Neu wird in diesem Jahr erstmals auch der beste Videoclip als Kategorie aufgenommen. Außerdem gibt es für die beste zahntechnische Meisterarbeit den Implantat-Prothetik-Preis, dotiert mit $2500 €$, der in $\mathrm{Zu}$ sammenarbeit mit der Klaus-Kanter-Stiftung ausgeschrieben und verliehen wird. Die Arbeiten dürfen nicht älter als 2 Jahre sein, die wissenschaftlichen Publikationen müssen in einem Journal mit Peer-Review-Verfahren veröffentlicht worden sein.Die PEERS-Förderpreise wurden ins Leben gerufen, um jüngere Wissenschaftler und wissenschaftlich interessierte

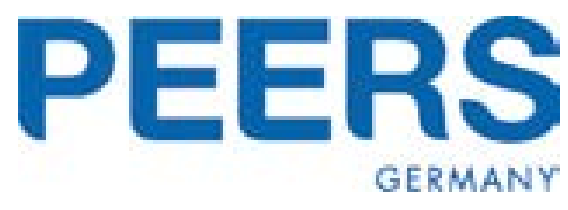

Zahnärztinnen und Zahnärzte in ihrer Arbeit zu unterstützen. Initiator der Preise ist PEERS (Platform for Exchange of Experience, Education, Research and Science), ein von DENTSPLY Implants unterstütztes internationales Expertennetzwerk mit Mitgliedern aus Klinik und Praxis sowie der Zahntechnik. Weitere Informationen zu den PEERS-Förderpreisen, Teilnahmebedingungen, eine Postervorlage und das Anmeldeformular können unter peers. foerderpreise@dentsply.com angefordert oder unter www.dentsplyimplants.de abgerufen werden. Die Arbeiten müssen bis zum 30. Juni 2016 eingereicht werden.

Nach einer Pressemitteilung der Dentsply Sirona Implants, Mannheim 